\title{
L'influence de la présentation multimodale d'items lexicaux sur la mémorisation en L2 (langue étrangère) chez des enfants de 8 à 11 ans
}

\author{
Marie-Ange Dat \\ Université de Nantes, CREN (EA 2661) \\ marie-ange.dat@univ-nantes.fr
}

\section{Position du problème}

L'apprentissage d'une langue étrangère par les enfants reste un domaine de recherche largement en construction. Les objectifs d'apprentissage qui concernent essentiellement la compréhension et l'expression de l'oral en L2, font partie des thèmes de recherche à privilégier. Le multimédia est un outil considéré par les jeunes apprenants comme attractif, et il est précisément pourvoyeur d'activités orales fondées sur des situations souvent fictives mais convaincantes, dans lesquelles les enfants sont invités à agir. Intégration de différents médias (écrit, image, son) proposés en interaction avec un utilisateur donné, le multimédia permet donc une présentation multimodale des contenus langagiers.

Mais la recherche dans ce domaine a essentiellement été menée «en laboratoire, indépendamment de (celle) concernant les apprentissages dans les contextes éducatifs » (Tergan, 1997 : 42). L'aspect que nous souhaitons ici mettre prioritairement en avant, réside dans l'aide au travail de mémorisation du lexique en L2 qu'il permet, car le vocabulaire peut être présenté sous de multiples formes, grâce à la multimodalité inhérente à l'outil informatique. Il reste à en évaluer les conditions. La psychologie cognitive justement, étudie notamment les processus mentaux en jeu dans le langage et la mémoire, processus de traitement des informations en vue de la meilleure adaptation possible à un environnement, la langue étrangère en l'occurrence (Gaonac'h, 2003). La compréhension, étape fondamentale de l'apprentissage présecondaire des langues étrangères, est une compétence dont le traitement intéresse fortement les sciences cognitives. Elle se conçoit comme un processus actif, composé de plusieurs stades successifs ou simultanés, qui passent de la mémoire à court terme à une interprétation sémantique complète. Et plus les traitements sont automatisés, moins le coût cognitif (Tricot, 1998 ; Sweller, 2003) engendré est élevé.

Pour poursuivre son apprentissage, l'élève qui se trouve en situation de compréhension va devoir solliciter son aptitude à la mémorisation. Les outils multimédias sont propices à la mémorisation du vocabulaire, mais les paramètres psycholinguistiques en jeu sont peu contrôlés. Ceci nous a incitée à nous pencher sur l'influence de la multimodalité sur la mémorisation du vocabulaire. On présente en effet souvent la multimodalité comme favorable à l'apprentissage compte tenu de l'impression positive que donne la présentation simultanée des textes et des images par rapport à un format unimodal. Pourtant, la charge cognitive engendrée peut être une explication aux difficultés de traitement de l'information, les formats de présentation pouvant constituer une charge trop grande. Les codages visuels et verbaux ne dépendent pas des mêmes structures et les formats de stockage en mémoire permanente en sont donc logiquement modifiés. Par conséquent, présenter à un sujet novice dans le domaine de connaissances où il est apprenant, une nouvelle information accompagnée d'une image, pourrait alourdir la charge cognitive en introduisant une difficulté supplémentaire : un dédoublement de l'attention.

\section{Les hypothèses}

Nous souhaitons donc évaluer les effets des combinaisons des modalités de présentation d'un vocabulaire en L2, proposé par l'outil informatique. Notre objectif vise à rendre compte de la présentation 
multimodale la plus efficiente en termes de mémorisation d'un lexique en L2 chez des enfants de 8 à 11 ans. Nous voulons vérifier l'impact de la modalité d'encodage (oral vs écrit vs combinaisons des deux) sur la mémorisation lexicale, selon une utilisation des modalités auditives et visuelles séquentielles (l'une après l'autre) ou simultanées (l'une en même temps que l'autre) : les mots de vocabulaire sont présents dans le matériel des tests à la fois en modalité auditive (mot entendu) et visuelle (mot lu).

Nous procèderons à partir d'une série de tests réalisés à l'aide d'une tâche de désignation ${ }^{1}$. Ces tests ont été effectués «en réponse immédiate» tout se suite après la phase d'exposition/apprentissage au vocabulaire, et «en réponse différée », une semaine après la phase d'exposition/apprentissage. Cette deuxième phase permet de rendre compte des connaissances mémorisées dans la mémoire à long terme. Désormais, nous désignerons comme performances, les bonnes réponses des enfants.

Notre recherche a été guidée par des hypothèses d'ordres différents, découlant du caractère exploratoire de notre démarche. Nous retiendrons ici les 3 principales.

Hypothèses 1) Nous avons d'abord souhaité évaluer l'influence de l'âge des participants sur la mémorisation et le temps d'exécution :

1a) Comme l'âge des élèves influence leurs ressources attentionnelles (plus ils sont âgés, plus ces ressources sont grandes) (Dempster, 1981; Swanson, 1999, cités par Gaonac'h et Fradet, 2003), les performances seraient plus élevées chez les participants les plus âgés.

1b) Puisque la maturité cognitive est proportionnelle à l'âge de l'enfant et que la rapidité d'exécution d'une tâche est liée à la maturité (Piaget et Inhelder, 1966), plus les participants sont âgés, plus court serait ce temps d'exécution.

Hypothèses 2) Nous avons ensuite centré nos interrogations sur l'influence de la présentation multimodale de la langue étrangère sur la mémorisation du lexique, chez des enfants de trois tranches d'âges compte tenu de l'hypothèse précédemment énoncée.

2a) Puisque l'écrit représente plus de difficulté que l'oral en L1 (Bourdin et Fayol, 1994), les participants de CE2 de niveau scolaire moyen obtiendraient des performances plus élevées avec la modalité d'encodage « oral » qu'avec l'encodage « écrit ».

2b) Il est communément admis que les modalités de présentation conjointe du lexique (écrit+oral) favorisent l'apprentissage (Kalyuga, Chandler et Sweller, 2000 ; Le Bohec et Jamet, 2002), affirmation reprise comme argument commercial de nombre de maisons d'édition, la double modalité de présentation simultanée de l'item («écrit+oral») faciliterait la mémorisation en comparaison d'une présentation unimodale, et occasionnerait des performances supérieures.

Hypothèse 3) Notre dernier questionnement porte sur l'influence de la transparence (L1 sur L2) sur la mémorisation : plus un mot est transparent, plus il est mémorisé (Lado, 1957; Giacobbe, 1992; Vogel, 1995 ; Alsulaimani, 1990, Teemant, 1988 et Laufer, 1991, cités par Théophanous, 2001), les performances seraient donc les meilleures pour les mots les plus transparents.

Les hypothèses que nous formulons nécessitent comme nous allons le voir, la mise en place d'un matériel d'expérimentation léger. Pendant les tests de désignation, l'enfant est seul devant l'ordinateur et les actions qu'il exerce sont effectuées par le biais de la souris, avec laquelle il désigne l'objet dénoté à l'oral (voix enregistrée et dite par la mascotte du jeu) et/ou à l'écrit (apparent à l'écran).

\section{Les tests de désignation}

L'expérimentation a été menée au sein d'une équipe de recherche pluridisciplinaire, financée par la Région Midi-Pyrénées, France ${ }^{2}$ (voir note de fin de document $\mathrm{n}^{\circ} 2$ ). Ce projet portait sur la mise en évidence des éléments présidant à la conception d'un cédérom d'accompagnement pour l'apprentissage d'une langue étrangère pour des adultes, ainsi qu'une méthode d'évaluation de cédéroms d'enseignement de langue étrangère. Une partie de cette recherche consistait à évaluer l'existant: un cédérom de 
l'entreprise Milan Presses partenaire financier du projet. C'est la raison pour laquelle l'étude a été menée à partir du logiciel « Mobiclic », magazine électronique de cette maison d'éditions.

Nous avons sélectionné le thème et la scène que nous allions intituler "L'île de tes rêves " parmi les différents numéros de ce magazine. Ce choix a été guidé par plusieurs critères : les lexies devaient désigner des objets concrets, fonctionnels ; ces objets devaient faire partie de l'univers de l'enfant.

\section{Le matériel}

La planche de dessins (la future scène informatisée) que nous allions utiliser devait tenir compte des formats de présentation suivants : une planche unique (pas de passage d'une planche à l'autre, comme c'est le cas dans certains numéros) ; la lexie devait apparaitre toujours sous le dessin correspondant ; à un dessin devait correspondre une seule lexie ; toutes les lexies devaient être des substantifs, singuliers, précédés d'un article indéfini; les dessins étaient de taille proportionnelle à la perspective de la scène représentée.

Le matériel que nous avons utilisé à ces fins compte l'influence de variables dont nous n'avons pas tenu compte mais qu'il nous semblerait intéressant d'aborder dans d'autres recherches : les éléments du décor autres que les dessins représentant les mots à mémoriser, la taille des icônes, leur disposition, l'animation dont certaines font l'objet, les couleurs utilisées.

Il nous importait aussi d'évaluer l'aspect prototypique de cette scène imagée conçue par un graphiste, afin de nous rendre compte à quel point le dessin était le reflet de l'organisation d'objets que des enfants s'attendent à voir lorsqu'ils activent mentalement un réseau sémantique à partir de l'input « L'île de tes rêves ». Nous nous référons ici aux travaux ayant trait à la catégorisation (Dubois, 2002) qui insistent sur le caractère individuel et culturellement marqué de l'organisation lexicale cognitive d'un individu.

Pour vérifier l'aspect prototypique et familier de la scène, nous avons mené une enquête auprès d'un groupe d'enfants de la même tranche d'âge que ceux qui devaient participer aux tests de désignation, soit auprès de 148 élèves de cycle 3 (de 8 à 11 ans) : deux classes de CE2 (43 participants, âge moyen : entre 8 et 9 ans), deux classes de CM1 (49 participants, âge moyen : entre 9 et 10 ans), et deux classes de CM2 (56 participants, âge moyen : entre 10 et 11 ans). Nous avons demandé à ces enfants d'écrire (de façon anonyme et même de manière très approximative car il ne s'agissait pas de tester leurs compétences en orthographe) les mots, les phrases (les dessins ont été acceptés) qui leur venaient à l'esprit quand on leur demandait de parler de l'île de leurs rêves.

Nous avons ensuite comparé les productions des élèves aux items figurant sur la planche imagée (voir en annexes) du numéro de « Mobiclic » retenu pour l'expérience. Puis, nous avons pu sélectionner les items pour notre expérience : sur les 12 finalement retenus, huit sont issus de cette enquête. Nous les avons gardés pour leur aspect prototypique : le cocotier / the coconut tree ; le coquillage / the shell ; le soleil / the sun ; le bateau / the boat; l'île / the island; le nageur / the swimmer; le nuage / the cloud; le trésor / the treasure. Les quatre autres items ont été peu ou pas du tout donnés par les enfants : le crabe / the crab; le rocher / the rock; le perroquet / the parrot; le serpent / the snake. Nous les avons cependant conservés du magazine d'origine compte tenu de leur occurrence acceptable dans la scène et de notre hypothèse sur la relation entre transparence de mot entre les langues (L1 et L2) et leur mémorisation. L'objet « serpent » nous a semblé intéressant à garder pour sa forme opaque et malgré sa présence un peu inattendue en ces lieux ainsi que sa non prégnance visuelle (au premier plan, au centre de la planche mais de la même couleur que les brins d'herbe).

La méthode

Les participants que nous avons testés sont 332 enfants de cinq écoles élémentaires considérées par l'Education nationale française et les enseignants comme socialement mixtes. Trois niveaux scolaires différents ont été testés à travers le cycle 3 (CE2, CM1, CM2, élèves de 8 à 11 ans) : 135 élèves de CE2 (âge moyen : de 8 à 9 ans), 102 élèves de CM1 (âge moyen : de 9 à 10 ans), 95 élèves de CM2 (âge moyen : de 10 à 11 ans). Les différences d'effectifs entre classes proviennent des niveaux de compétences scolaires recherchés et testés. Nous avons ainsi indiqué aux enseignants (professeurs des écoles) les deux 
profils d'élèves précis que nous recherchions et que nous appellerons pour simplifier « de niveau moyen » et «en facilité scolaire» afin de constituer des échantillons homogènes. Les critères qui nous ont permis d'établir ce classement et ces appellations reposent sur les évaluations réalisées en milieu scolaire par les enseignants des élèves participants, soumis aux instructions officielles de leur ministère, ainsi que sur les résultats des évaluations nationales d'entrée au CE2, qui présentent l'avantage de garantir des conditions et des contenus d'évaluation de qualité équivalente pour tous les élèves.

Comme les enfants testés étaient en première année d'apprentissage d'une langue étrangère, aucun critère définitoire relatif à cette matière n'a été établi. Au moment des tests, tous les apprenants étaient de niveau A1.1 selon le CECR (Cadre européen commun de référence pour les langues). L'anglais a été choisi parce que c'est la langue la plus fréquemment enseignée en cycle 3.

\section{Plan d'expérience}

Cinq modalités d'encodage des items lexicaux ont été déterminées pour évaluer la mémorisation : « oral », « écrit », « oral+écrit simultanés », « oral puis écrit », « écrit puis oral », ainsi que 2 modalités de désignation : orale (l'enfant entend l'item et le désigne par un clic) et écrite (l'enfant lit l'item et le désigne par un clic).

\section{Procédure}

Les élèves sont passés individuellement et 2 phases leur ont été demandées :

- une phase de test passée immédiatement après un temps d'apprentissage (durée d'environ 10 minutes selon la rapidité de réponse aux tests des participants) qui fait suite à une phase de démonstration par la mascotte du jeu, et qui était rappelée par l'examinateur;

- une phase de test passée en différé d'une semaine, sans la phase d'apprentissage (2 minutes 30 environ selon la rapidité de réponse aux tests des participants).

Les deux tests (immédiat et différé) demandés aux enfants ont été passés dans la même modalité, celle qui a été sélectionnée au départ.

Pendant la phase d'apprentissage, le participant peut faire glisser la souris et cliquer autant qu'il le souhaite, dans l'ordre de son choix. Chaque objet survolé se met en surbrillance pour ne pas être oublié. La désignation de l'objet est bien sûr donnée par la mascotte du jeu selon la modalité choisie lors de la phase de sélection, et s'effectue de la même façon que pendant la phase de démonstration.

Au bout des 5 minutes de la phase d'apprentissage, l'application lance le premier mot annoncé par un bip sonore (tirage aléatoire de 1 sur 12). Pour le deuxième tirage et les suivants, on soustrait le(s) tirage(s) déjà effectué(s). L'enfant dispose de 17 secondes pour répondre. Le clic détermine la fin de l'action et lance le mot suivant. S'il n'y a pas de réponse au bout des 17 secondes, un nouveau mot est annoncé par un bip sonore. Afin d'éviter le plus possible les réponses hasardeuses, il est bien indiqué au participant qu'il doit cliquer sur l'icône «Je ne sais pas » à droite de la planche animée, pour chaque mot non mémorisé.

Les 12 mots passés, la mascotte réapparaît et annonce le score à l'enfant. Elle propose aussi un corrigé : chaque objet du dessin se met un à un en surbrillance tandis que la désignation de l'objet est donnée selon la modalité sélectionnée au départ. Les enfants sont invités à la regarder attentivement puis la mascotte les remercie. L'activité est enregistrée dans le dossier généré en début de tests.

\section{Les résultats}

Les résultats obtenus sont issus des 664 fichiers informatisés (332 participants x 2 passages) soit 7968 données (664 fichiers x 12 items) soumis à des traitements statistiques correspondant à une analyse de la variance (variation et comparaison des facteurs de l'expérience). Ces résultats proviennent des variables suivantes : les 12 mots à mémoriser, les 3 classes/âges, les 2 niveaux scolaires pour les élèves de CE2 (en 
facilité scolaire vs niveau moyen), les 5 modalités d'encodage, les 2 modalités de désignation, les 2 moments de réponse, immédiate vs réponse différée d'une semaine.

L'intégralité des traitements statistiques présentés par hypothèse est tenue à la disposition du lecteur, nous ne présenterons ici que les différences (Dat, 2006). Quand aucune précision n'est apportée, les données statistiques proposées sont conformes au seuil conventionnel de significativité de 0.05 .

Par le premier volet de nos hypothèses, nous avons souhaité évaluer l'influence de l'âge des participants sur la mémorisation et le temps d'exécution de la tâche.

L'hypothèse 1 est ici confirmée par les résultats qui montrent que conformément à nos attentes, plus l'âge des participants est élevé, plus les performances augmentent, toutes modalités d'encodage confondues. Tant pour les réponses immédiates que pour les réponses différées, le facteur âge intervient sur les performances, avec une $\mathrm{p}$ valeur $=0$ et un facteur d'inflation de la variance (FIV, qui traduit la façon dont un facteur est lié aux autres) $<5$ (1,3 pour l'ensemble des participants, tant en réponse immédiate que différée). Les performances en réponse immédiate des élèves de CM1 (âge moyen : de 9 à 10 ans) sont supérieures de 1,67 par rapport à celles de CE2 (âge moyen : de 8 à 9 ans) classe de référence ici, tandis que les performances des élèves de CM2 (âge moyen : de 10 à 11 ans) sont supérieures de 0,95 par rapport à celles des CM1, et de 2,62 par rapport aux performances des élèves de CE2.

Les performances en réponse différée des élèves de CM1 sont supérieures de 1,35 par rapport à celles de $\mathrm{CE} 2$, tandis que les performances des élèves de CM2 sont supérieures de 1,08 par rapport à celles des CM1, et de 2,43 par rapport aux performances des élèves de CE2.

En outre, plus les enfants sont âgés, plus court est le temps d'exécution. Conformément à nos attentes, tant pour les réponses immédiates que pour les réponses différées, le facteur «temps » par rapport à l'âge/la classe des participants intervient, avec une $\mathrm{p}$ valeur inférieure ou égale à 0,012 pour les réponses immédiates et égale à 0 pour les réponses différées des participants de CM2, mais une $\mathrm{p}$ valeur non significative pour les réponses différées des participants de CM1 $(0,21)$. Le facteur d'inflation de la variance (FIV) reste nettement $<5 \quad(1,3$ pour l'ensemble des élèves tant en réponse immédiate que différée).

Les temps moyens de réponse immédiate des élèves de CM1 (âge moyen : de 9 à 10 ans) sont inférieurs de 0,372 par rapport à ceux de CE2 (âge moyen : de 8 à 9 ans) classe de référence ici encore, tandis que les temps des élèves de CM2 (âge moyen : de 10 à 11 ans) sont inférieurs de 0,551 par rapport à ceux des élèves de CM1, et de 0,923 par rapport aux temps moyens de réponse immédiate des élèves de CE2.

Les temps moyens de réponse différée des élèves de CM1 sont inférieurs de 0,171 par rapport à ceux de $\mathrm{CE} 2$, tandis que les temps des élèves de CM2 sont inférieurs de 0,768 par rapport à ceux des élèves de CM1, et de 0,939 par rapport aux temps moyens de réponse différée des élèves de CE2.

Le test $t$ de Student suivant complète ces résultats en nous donnant la moyenne des temps de réponse immédiate (RI) et celle des temps de réponse différée (RD). La moyenne en réponse immédiate n'est supérieure que de 0,11 par rapport à la moyenne en réponse différée.

Le deuxième volet de nos hypothèses a trait à l'influence d'une présentation multimodale du lexique en langue étrangère sur la mémorisation.

L'hypothèse 2 a («puisque l'écrit représente plus de difficulté que l'oral en L1 (Bourdin et Fayol, 1994), les participants de CE2 de niveau scolaire moyen obtiendraient des performances plus élevées avec la modalité d'encodage « oral » qu' " écrit») est infirmée par les résultats obtenus par le biais d'analyses de régression. Les résultats obtenus sont significatifs ( $\mathrm{p}$ valeur $=0,042$ en réponse immédiate, et 0,013 en réponse différée). Le facteur d'inflation de la variance (FIV) est très nettement inférieur à 5 (1,6 en réponse immédiate et 1,1 en réponse différée).

Ces résultats nous montrent qu'en réponse immédiate, les performances de la modalité d'encodage « oral » (seul) sont inférieures de 2,70 par rapport aux résultats de la modalité d'encodage « écrit » (seul), modalité de référence ici. 
En réponse différée, les performances de la modalité d'encodage «écrit» (seul) augmentent encore et sont supérieures de 2,77 à la modalité d'encodage « oral» (seul), modalité de référence de cette deuxième analyse de régression.

Des données soumises à une analyse de variance multivariée de l'ensemble de la population (et non plus seulement les élèves de CE2), présentent un effet statistiquement significatif de la variable «niveau scolaire » $(p$ valeur $=0,04)$ : les élèves de «niveau moyen » ont des performances moins élevées que ceux « en facilité scolaire » quelle que soit la modalité d'encodage.

Nous constatons aussi un effet statistiquement significatif d'interaction entre les variables «niveau scolaire » et «modalité d'encodage» ( $p$ valeur $=0)$ : la modalité «oral» met plus spécifiquement en difficulté les élèves de «niveau moyen », néanmoins, avec la modalité « écrit puis oral » l'effet disparaît, leurs performances atteignent quasiment le niveau des élèves « en facilité scolaire ».

L'hypothèse 2b) («Il est communément admis que les modalités de présentation conjointe du lexique (écrit+oral) favorisent l'apprentissage (Kalyuga, Chandler et Sweller, 2000 ; Le Bohec et Jamet, 2002), affirmation reprise comme argument commercial de nombre de maisons d'édition, la double modalité de présentation simultanée de l'item ("écrit+oral») faciliterait la mémorisation en comparaison d'une présentation unimodale, et occasionnerait des performances supérieures») n'est elle non plus pas confirmée dans le cas de la présentation simultanée des modalités d'encodage.

Ces traitements donnent lieu à des résultats significatifs $(\mathrm{p}$ valeur $=0)$ pour l'ensemble des données traitées, notamment pour les modalités d'encodage concernées : «oral » (seul) (encodage de référence dans le cadre des traitements), « écrit» (seul) et oral+écrit (simultanés). Le FIV (facteur d'inflation de la variance) reste bas puisque bien inférieur à 5 (inférieur à 1,5).

Par cette analyse, nous constatons que pour les tests immédiats, la modalité d'encodage " oral+écrit » (simultanés) est supérieure de 2,59 par rapport à la modalité d'encodage «oral» (seul), mais que la modalité d'encodage «écrit» (seul) lui est supérieure de 3,80, ce qui donne une différence de 1,21 en faveur de cette modalité.

Les tests différés présentent une différence moins accentuée $(2,06-2,82=0,76)$, mais persistante après une semaine, toujours en faveur de la modalité d'encodage «écrit» (seul). La modalité d'encodage « oral » seul reste celle qui occasionne le taux de performances le plus bas.

Nous constatons un effet statistiquement significatif d'interaction entre la classe et la modalité d'encodage $(p=0,002)$ pour les réponses immédiates, et $(p=0)$ pour les réponses différées.

Enfin, nous souhaitions par l'hypothèse 3) vérifier l'effet de la transparence phonétique et graphémique de la L1 sur la mémorisation du lexique en L2. En effet, plus un mot est transparent, plus il est mémorisé (Lado, 1957 ; Giacobbe, 1992 ; Vogel, 1995 ; Alsulaimani, 1990, Teemant, 1988 et Laufer 1991, cités par Théophanous, 2001), les performances seraient donc les meilleures pour les mots les plus transparents.

Nous avons fait appel dans un premier temps aux statistiques descriptives pour obtenir le classement des mots les mieux mémorisés dans ce contexte en «réponse immédiate vs réponse différée », toutes variables confondues. En réponse immédiate et par ordre décroissant, les mots les mieux mémorisés sont : crab; coconut tree ; rock ; island ; treasure ; parrot ; boat ; swimmer ; snake ; shell ; sun ; cloud.

En réponse différée, et par ordre décroissant, les mots les mieux mémorisés sont présents sensiblement dans le même ordre : crab; coconut tree; rock; treasure; island; parrot; boat; swimmer; sun; snake; shell; cloud. 


\section{Discussion}

Par notre première hypothèse, nous avons d'abord souhaité considérer l'influence de l'âge sur la mémorisation et le temps d'exécution.

Les âges des participants s'échelonnent de 8 à 11 ans et se retrouvent répartis en trois classes scolaires : $\mathrm{CE} 2, \mathrm{CM} 1$, et CM2. Cette répartition en âge met en évidence qu'un enfant de début d'année peut se retrouver dans la même classe qu'un enfant de fin d'année, par conséquent, c'est le facteur « classe » qui semble être le plus pertinent à prendre en compte. De fait, l'effet statistiquement significatif du facteur «classe» indique que les participants qui sont dans des niveaux de classe élevés ont de meilleures performances, tant en réponse immédiate que différée.

Les performances en réponse différée baissent peu par rapport aux performances en réponse immédiate : $+1,67$ pour les élèves de CM1 par rapport aux CE2; + 2,62 pour les élèves de CM2 en réponse immédiate contre $+1,35$ et $+2,43$ en réponse différée.

Cela implique que l'effet de niveau de classe a aussi des incidences sur la mémorisation à long terme, ici une semaine après la phase d'apprentissage des 12 mots. Les performances des élèves de CM2 s'affichent parmi les meilleures (supérieures à 6/12) alors que toutes les performances inférieures ou égales à 6/12 proviennent d'élèves de CE2. Les 8 mots les moins mémorisés l'ont été par des élèves de cet âge (entre 8 et 9 ans). Dans la même logique, les performances des CM1 se situent majoritairement (10/12) dans des positions intermédiaires.

Les résultats sont fortement liés à la classe des participants, ce qui correspond aux conclusions de plusieurs travaux en L1. La mémoire de recognition qui apparaît très tôt (Piaget et Inhelder, 1966) s'affine quand l'âge des enfants augmente. Les enfants plus âgés recodent plus facilement les stimuli visuels (Gaonac'h et Fradet, 2003), ce qui concourt à améliorer leurs performances. Des recherches menées par Miles, Morgan, Milne et Morris (1996, cités par Gaonac'h et Fradet, 2003) avancent que dès 7 ans, les représentations visuo-spatiales augmentent, ce qui renforce l'empan mnémonique. Les éléments verbaux que les enfants peuvent rappeler sont environ au nombre de 6 à 9 ans, pour des performances qui atteignent celles des adultes vers 11-12 ans (Dempster, 1981, cité par Gaonac'h et Fradet, 2003). L'effet de la scolarisation corroboré à celui de l'âge permet d'avancer que les exercices de jeux de mémoire sont à encourager (Gaonac'h et Fayol, 2003).

Plus les participants sont âgés, plus leurs réponses sont rapides (Hypothèse 1b). L'effet du facteur « classe » est significatif pour tous les résultats, à l'exception des performances de la classe de CM1 en différé dans l'analyse de variance. Nous savons pour la modalité d'encodage « oral » que la création des images sonores nécessaires à la représentation des stimuli oraux en mémoire progresse au fur et à mesure du développement de l'enfant (Piaget et Inhelder, 1966), et que pour l'encodage « écrit », plus le lecteur est confirmé plus le traitement des mots nouveaux qu'il effectue est automatisé et donc rapide (Fayol, 2005 ; Gombert, 2005). Ces éléments influencent certainement les temps de réponse des participants de notre expérimentation. Les travaux de Palmer (2000, cité par Gaonac'h et Fradet, 2003) indiquent que les très jeunes enfants n'utiliseraient pas de stratégies de rappel mais les deux stratégies de codage de l'information, visuelle et verbale : il semble alors logique que les plus jeunes des participants de nos tests soient ralentis par l'utilisation de ces deux stratégies.

Il nous semble intéressant de souligner le maintien des temps de réponse moyens entre la désignation du lexique immédiatement après la phase d'apprentissage et celle qui a eu lieu une semaine après. La totalité des temps de réponse a peu augmenté, alors que, rappelons-le, la deuxième phase du test a lieu sans nouvel apprentissage. La mémorisation à long terme a conservé l'apprentissage du jeu de mémoire. Le modèle de compréhension en L2 de Nagle et Sanders (1986) pose que les données traitées par la mémoire à court terme sont transférées en mémoire à long terme une vingtaine de secondes après ce traitement. Ceci expliquerait le peu de variation qui intervient entre les performances en réponse immédiate et en réponse différée.

A cause de la lourdeur du traitement cognitif de l'écrit pour les enfants qui sont apprentis lecteurs/scripteurs ou en difficulté face à ces compétences, nous nous attendions à voir de meilleures 
performances à l'oral chez les plus jeunes des participants et chez ceux évalués en niveau moyen par l'institution scolaire (hypothèse 2a). En effet, nous savons que chez les lecteurs débutants en L1: a) le manque de fréquentation des mots (les mots nouveaux donc) ne permet pas les inférences inconscientes facilitantes (Fayol, 2005) et l'accès aux éléments sémantiques en mémoire à long terme (Bourdin et Fayol, 1994); b) la décomposition séquentielle du mot nouveau en segments de taille variable est une activité coûteuse (Berent et Perfetti, 1995; Fayol, 2005); c) sans l'influence de mots connus, la reconnaissance des mots nouveaux est rendue difficile (Frith, 1985); et d) la reconnaissance des morphèmes se fait tardivement (Gombert, 2005).

En outre, certaines données concernant la mémorisation pouvaient appuyer cette hypothèse : nous savons que la nature de l'encodage d'un mot détermine la qualité de son rappel en mémoire. Plus le traitement du mot à rappeler est profond meilleure sera sa probabilité de rappel, et la référence au sens permet un meilleur traitement qu'une référence au son, elle-même meilleure qu'une référence visuelle. (Craik et Lockhart, 1972, cités par Baddeley, 1992 ; Cordier et Gaonac’h, 2004).

Or, nos résultats semblent montrer que pour la lecture de lexique en L2, la modalité d'encodage « oral » n'apporte pas l'étayage attendu en termes de mémorisation sur notre échantillon d'élèves de CE2 « de niveau moyen ». En réponse immédiate, cette modalité est plus faible de 2,70 par rapport à la modalité « écrit », et le résultat est plus bas encore (de 2,77) en réponse différée.

$\mathrm{Si}$ les didacticiens des langues étrangères savent que la prononciation des apprenants est meilleure lorsqu'ils acquièrent le lexique oralement, la mémorisation semble cependant être largement favorisée par une présentation écrite. Nous retrouverons ce résultat sur toute la population et pas seulement chez les participants de niveau scolaire moyen, même si l'effet est, pour cette population, plus fort. Les processus de mémorisation semblent donc différer quand un enfant de 8 ans doit traiter du lexique en L1 et en L2. En L1, la modalité orale faciliterait le traitement, et en L2, l'écrit représenterait un appui, comme pour les participants de notre expérience. Ces résultats montrent que par rapport à la tâche demandée, la mémorisation visuelle est plus utilisée par le jeune apprenant que la mémorisation auditive. L'écrit représente ici cet étayage visuel.

La multimodalité est souvent annoncée comme facteur favorisant l'apprentissage des langues (Kalyuga, Chandler et Sweller, 2000 ; Le Bohec et Jamet, 2002) (Hypothèse 2b). Nous pouvions par conséquent nous attendre à ce que la modalité d'encodage "oral+écrit » (simultanés) permette des performances de mémorisation plus élevées que celles enregistrées avec des stimuli unimodaux (« oral» (seul) et «écrit» (seul)). Or, nous constatons des performances plus faibles dans les tests avec la double modalité que celles obtenues avec l'encodage " écrit» (seul). Compte tenu des résultats des hypothèses précédentes, on pourrait dire que le son dans ce contexte serait traité avant l'écrit et représenterait un alourdissement de la charge cognitive pour le traitement de l'information.

Les performances sont néanmoins plus importantes que celles correspondant à la modalité d'encodage «oral» (seul). Ceci indique que la modalité «écrit» donne accès ici encore à de meilleurs taux de mémorisation, et qu'elle n'est pas soutenue par la présentation conjointe de l'oral. En revanche, les deux modalités présentées l'une après l'autre («oral puis écrit»; «écrit puis oral») permettent des performances supérieures aux trois précédentes : la double modalité de présentation joue dans ce cas un rôle d'étayage pour la mémorisation lexicale.

Ces résultats vont dans le même sens que les éléments avancés par la théorie du double codage de Clark et Paivio (1991) : deux sources d'information présentées simultanément (texte, images) ne facilitent pas la mémorisation si l'une des deux requiert une grande partie ou la totalité des ressources attentionnelles. Dans les tests de désignation dont nous parlons, nous pensons que la modalité d'encodage «oral»a un coût de traitement cognitif tel, qu'elle mobilise une grande partie de l'attention de l'enfant, et qu'elle reste handicapante dans le cas de la modalité «oral+écrit» simultanés. Le traitement de l'input oral mobiliserait les ressources attentionnelles de l'enfant débutant en L2 et perturberait les traitements plus aisés de l'écrit. En effet, en nous référant au principe de contiguiité, à savoir l'effet de la présentation simultanée des textes et des images sur la construction des connaissances (Mayer et Moreno, 1998), notre interprétation des résultats semble plausible. Pour que deux sources d'information présentées (texte, 
images) constituent une aide en termes de mémorisation, aucune des deux ne doit requérir une plus grande partie des ressources attentionnelles du sujet.

La présentation conjointe de deux modalités d'encodage («oral » et «écrit») d'un support imagé ne favorise donc pas en soi la mémorisation du lexique présenté en L2.

Les mots donnés à apprendre aux participants des tests peuvent être définis comme plus ou moins transparents en langue anglaise par rapport à la langue française, L1 des participants. Selon les travaux cités en 2) (Lado, 1957; Giacobbe, 1992 ; Vogel, 1995 ; Alsulaimani, 1990, Teemant, 1988 et Laufer 1991, cités par Théophanous, 2001), nous avons émis l'hypothèse que plus un mot est transparent, meilleure serait sa mémorisation (hypothèse 3).

Les résultats présentés nous indiquent l'ordre moyen des désignations des 12 mots, tous participants confondus. En réponse immédiate, du plus désigné au moins désigné : crab; coconut tree ; rock ; island ; treasure ; parrot ; boat ; swimmer ; snake ; shell ; sun ; cloud.

Et en réponse différée : crab; coconut tree; rock; treasure; island; parrot; boat; swimmer; sun; snake; shell; cloud.

Admettre qu'un mot en L2 est transparent par rapport à un autre mot en L1 implique d'une part que les deux formes renvoient à une signification identique et d'autre part qu'au niveau perceptif (auditif ou visuel) il y ait de fortes ressemblances. Or, le français et l'anglais sont reconnus comme deux langues à l'orthographe irrégulière et opaque (Bourdin et Fayol, 1994). Par ailleurs, nous avons rappelé que la connaissance des correspondances grapho-phonologiques (appariements) de la langue française ne conduirait qu'à $50 \%$ de lexique retranscrit (Véronis, 1988), la forme orthographique étant liée à la mémorisation (Fayol, 2005). De plus, il a été mis en évidence que les lecteurs débutants notamment, procédaient par comparaison interlangue et comparaient donc les analogies entre la L1 et la L2 (Goswani, Gombert et Fraca de Barrera, 1998). Un effet de voisinage intervient par conséquent particulièrement entre le français et l'anglais, car plus l'orthographe est irrégulière, plus cet effet est marqué afin de faciliter la lecture.

Les représentations linguistiques les plus proches du français sont ainsi les mieux mémorisées : crab en tête de classement en est un exemple éloquent, de par une transparence quasi complète tant sur les plans phonétique que graphémique. Avec lui, rock, treasure et coconut tree (à la morphologie particulière) offrent un taux de désignation élevé tant en réponse immédiate que différée. Nous pouvons aussi rapprocher le bon résultat de désignation de coconut tree du fort taux de production spontanée correspondant, lors de l'enquête «L'île de tes rêves ».

Nous savons que la longueur du mot (son temps de prononciation) influence la mémorisation (Baddeley, 1992), or les résultats mitigés à ce stade, ne nous permettent pas d'explorer davantage ce point. Il aurait fallu intégrer à notre matériel davantage de mots courts, plus et moins transparents. La transparence de l'item en langue étrangère par rapport à son équivalent en langue première semble ainsi influencer la mémorisation.

\section{Références bibliographiques}

Baddeley, A.D., (1992). La mémoire humaine : théorie et pratique. Traduit sous la direction de Hollard, S., (édition originale Lauwrence Erlbaum Associates Ltd. Publishers, 1990), Grenoble: Presses Universitaires de Grenoble.

Berent, I., Perfetti, C.A., (1995). A rose is a REEZ : the two cycles models of phonology assembly in reading English. Psychological review, 102, 146-184.

Bourdin, B., Fayol, M., (1994). In Written Language Production More Difficult Than Oral Language Production? A Working Memory Approach. In International Journal of Psychology, vol. 29, n5, 591-620. 
Clark, J.M., Paivio, A., (1991). Dual Coding Theory and Education. In Educational psychology review, 3, 149-210.

Cordier, F., Gaonac'h, D., (2004). Apprentissage et mémoire. Paris : Nathan Université.

Dat, M.-A., (2006), Didactique présecondaire des langues étrangères : l'influence de la présentation multimodale du lexique sur la mémorisation chez des enfants de 8 à 11 ans. Thèse de doctorat des sciences du langage, Université Toulouse 2-Le Mirail.

Dat, M.-A., Spanghero-Gaillard N., (2007). Les implications de supports multimédias dans l'apprentissage du vocabulaire en L2. 2ème colloque international de didactique cognitive DIDCOG « Français (langue étrangère/ langue seconde/ langue maternelle) », Université Toulouse 2 - Le Mirail, 19-21 septembre.

Dubois, D., (2002). Les mots de la perception : cultures, connaissances, et cognitions. Les ateliers de l'ANVIE, $\mathrm{n}^{\circ} 25$.

Fayol, M., (2005). Apprendre l'orthographe Le cas du français. In Billières, M., Spanghero-Gaillard, N., (dir.), Revue Parole numéro spécial, 34-36.

Frith, U., (1985). Beneath the Surface of Developmental Dyslexia. In Patterson, K.E., Marshall, J.C., Coltheart, M., (Eds.), Surface dyslexia: Cognitive and Neuropsychological Studies of Phonological Reading, Lawrence Erlbaum, Hillsdale.

Gaonac'h, D., (2003). Comprendre en langue étrangère. In Gaonac'h, D., Fayol, M. (dir.), Aider les élèves à comprendre/Du texte au multimédia. Paris : Hachette Education, 137-155.

Gaonac'h, D., Fayol, M., (dir.), (2003). Aider les élèves à comprendre/Du texte au multimédia. Hachette Education, Paris.

Gaonac'h, D., Fradet, A., (2003). La mémoire de travail : développement et implication dans les activités cognitives. In Kail, M., Fayol, M., Les sciences cognitives et l'école. Paris : PUF, 91-150.

Giacobbe, J., (1992). Acquisition d'une langue étrangère. Paris : CNRS Editions.

Gombert, J.E., (2005). Et si l'automatisation n'existait pas ? L'implicite et l'explicite dans l'apprentissage de l'écrit et ses troubles. In Billières, M., Spanghero-Gaillard, N., (dir.), Revue Parole numéro spécial, 34-36.

Goswami, U., Gombert, J.E., Fraca de Barrera, L. (1998). Children's Orthographic Representations and Linguistic Transparency: Nonsense word reading in English, French and Spanish. In Applied Psycholinguistics, vol.19, 19-52.

Kalyuga, S., Chandler, P. et Sweller, J. (2000). Incorporating Learner Experience into the Design of Multimedia Instruction. In Journal of Educational Psychology, 92, 126-136.

Lado, R. (1957). Linguistics across Cultures, applied Linguistics Language Teacher, Ann Arbor, University of Michigan Press.

Le Bohec, O., Jamet, E. (2002). Redondance verbale et niveaux de traitement. In Tricot, A., Molinier, P., (dir.), Compréhension et hypermédia, Actes du colloque d'Albi Centre Universitaire Champollion, 10 et 11 octobre, 91-107.

Mayer, R.E. Moreno, R., (1998). A Split-attention Effect in Multimedia Learning: Evidence for Dual Processing Systems in Working Memory. In Journal of Educational Psychology, vol.90, n². 312320.

Nagle, S.J. Sanders, S.L., (1986). Comprehension Theory and second language pedagogy. TESOL Quaterly, 20.1.

Piaget, J. Inhelder, B., (1966). La psychologie de l'enfant. Paris : PUF. 
Sweller, J. (2003). Evolution of Human Cognitive Architecture. In B.H. Ross (Ed.), The psychology of learning and motivation, 43, New York : Academic Press, 215-266.

Tergan, S.O. (1997). Misleading Theoretical Assumptions in Hypertext/Hypermedia Research. In Journal of Educational Multimedia and Hypermedia, vol.6, n³/4, 257-283.

Théophanous, O. (2001). Confusions lexicales dans l'acquisition du vocabulaire d'une langue secondelétrangère, Thèse de doctorat, Université de Montréal.

Tricot, A. (1998). Charge cognitive et apprentissage. Une présentation des travaux de John Sweller. In Revue de Psychologie de l'Éducation, pp.37-64, n³.

Véronis, J. (1988). From Sound to Spelling in French: Simulation on a Computer. In Cahiers de Psychologie Cognitive, 8, 315-334.

Vogel, K. (1995). L'interlangue, la langue de l'apprenant, traduit par Confais, J.P., et Brohee, J.M., Toulouse : PUM. 


\section{Annexes}

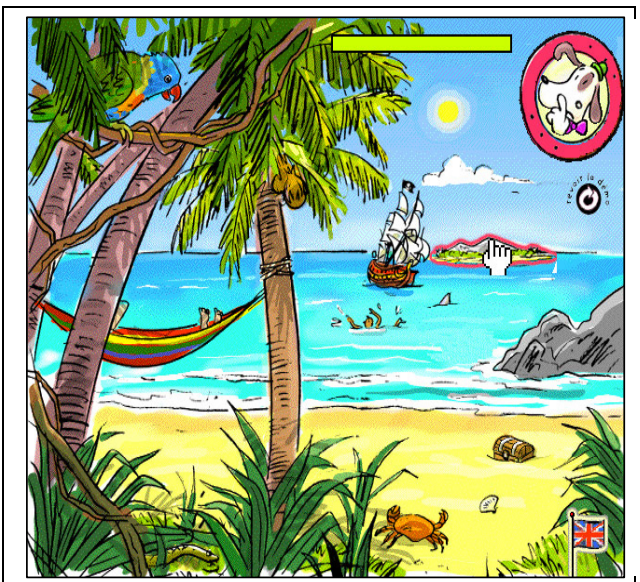

Scène informatisée « L'île de tes rêves » présentée aux enfants pour les tests

\footnotetext{
${ }^{1}$ Nous avons aussi (Dat \& Spanghero-Gaillard, 2007) procédé à une série de tests complémentaires menés dans un contexte équivalent, à partir d'une tâche de restitution (lexique dit et écrit).

${ }^{2}$ L'équipe de recherche était composée de quatre laboratoires toulousains : le laboratoire Jacques-Lordat, le Laboratoire Travail et Cognition (LTC), l'Institut de Recherche en Informatique de Toulouse (IRIT) et le CERFI (Centre d'études, de recherche sur la formation, d'innovation), laboratoire de l'IUFM (Institut universitaire de formation des maîtres). Cette équipe a rassemblé des didacticiens (langues étrangères et FLE/S), des linguistes et neuropsycholinguistes, des chercheurs en psychologie cognitive et en interaction humain-machine, ainsi que des ingénieurs de conception de systèmes interactifs et d'outils multimédias
} 